\title{
Variance in Clinical Profile and Use of Anticoagulants in Valvular and Non Valvular Atrial Fibrillation
}

\section{Smriti Shakya*, Arun Sayami, Ratna Mani Gajurel, Chandra Mani Poudel, Hemant Shrestha, Surya Devkota, Sanjeev Thapa, Rajaram Khanal}

Department of Cardiology, Manmohan Cardiothoracic Vascular and Transplant Centre (MCVTC), Institute of Medicine, TUTH, Kathmandu, Nepal

Email: ^drsmritishakya@gmail.com

How to cite this paper: Shakya, S., Sayami, A., Gajurel, R.M., Poudel, C.M., Shrestha, H., Devkota, S., Thapa, S. and Khanal, R. (2020) Variance in Clinical Profile and Use of Anticoagulants in Valvular and Non Valvular Atrial Fibrillation. World Journal of Cardiovascular Diseases, 10, 488-499. https://doi.org/10.4236/wjcd.2020.107049

Received: June 29, 2020

Accepted: July 24, 2020

Published: July 27, 2020

\section{Copyright $\odot 2020$ by author(s) and} Scientific Research Publishing Inc. This work is licensed under the Creative Commons Attribution International License (CC BY 4.0).

http://creativecommons.org/licenses/by/4.0/

(c) (i) Open Access

\begin{abstract}
Introduction: Atrial fibrillation is the most common cardiac arrhythmia encountered in clinical practice that affects morbidity and mortality to a large extent. This study was intended to determine various clinical profile and etiological factors in valvular and non-valvular atrial fibrillation and evaluate the usage of anticoagulants in them in the settings of developing nation like ours. Methods: A hospital based cross-sectional observational prospective study was conducted at Manmohan Cardiothoracic Vascular and Transplant Center, Institute of Medicine, Nepal for a period of sixteen months. All the patients with atrial fibrillation who were admitted in the cardiology department were included. The demographic profile, etiology, clinical features and the usage of anticoagulants were recorded. Results: A total of 175 patients with atrial fibrillation were enrolled in the study with $62 \%$ nonvalvular and $38 \%$ valvular cases. The overall mean age was $60 \pm 16.79$ years but among valvular atrial fibrillation, it was $47.9 \pm 14.013$ and among nonvalvular atrial fibrillation, it was $67.85 \pm 13.507$ years. Majority of patients were female (56\%). Sixty percent were from outside Kathmandu valley. Rheumatic heart disease was the most common cause $(38.9 \%)$ followed by dilated cardiomyopathy, systemic hypertension, degenerative valvular heart disease, coronary artery disease, etc. The most common presentation was dyspnea. About 79\% of valvular and $17 \%$ of nonvalvular atrial fibrillation patients were using anticoagulants. Conclusion: Atrial fibrillation was common in young females and rheumatic heart disease was the leading cause. There was inadequate use of anticoagulants in both valvular and nonvalvular atrial fibrillation probably due to the economic constraints and geographical hurdles.
\end{abstract}




\section{Keywords}

Atrial Fibrillation, Clinical Profile, Anticoagulation

\section{Introduction}

Atrial Fibrillation (AF) is a supraventricular tachyarrhythmia which shows uncoordinated atrial activation with ineffective atrial contraction and an electrocardiographic characteristic of irregular R-R intervals and absence of distinct $\mathrm{P}$ waves. It is a common cardiac arrhythmia which increases in prevalence with advancing age [1].

The overall prevalence of AF in western countries is $1 \%-2 \%$ of the general population [2]. The lifetime risks for development of AF are 1 in 4 for men and women 40 years of age and older [3]. The risk factors for AF are aging, male gender, hypertension, valvular disease, left ventricular dysfunction, hyperthyroidism and alcohol consumption [4]. The Lone AF or idiopathic AF accounts for $10 \%-15 \%$ of cases [5].

Atrial fibrillation is commonly classified as Valvular and Nonvalvular. The AF that occurs in the absence of rheumatic mitral stenosis, a mechanical or bioprosthetic heart valve, or mitral valve repair is considered Nonvalvular Atrial Fibrillation (NVAF) [1]. Profound studies are done in western countries which focus on NVAF due to low prevalence of rheumatic heart disease (RHD). However, the studies from Africa, Asia and the Middle East found that RHD is one of the commonest causes of atrial fibrillation accounting for $15 \%-60 \%$ of total AF cases [6] [7] [8]. The clinical significance and prompt treatment of AF is imperative due to its thromboembolic phenomenon that includes fatal stroke as well as due to heart failures which increase morbidity and mortality. The rate of ischemic stroke that occurs in patients with nonvalvular $\mathrm{AF}$ averages $5 \%$ per year, which is 2 to 7 times that of people without AF [9]. In patients with RHD and AF in the Framingham Heart Study, stroke risk was increased to 17 fold compared with age-matched controls, with the attributable risk of 5 times greater than those with non-rheumatic AF [10]. The presence of significant mitral regurgitation is associated with a lower risk of thromboembolism as compared to Rheumatic mitral stenosis [11] [12]. Oral anticoagulants (OAC) reduce the risk of stroke by $64 \%$ and all-cause mortality by $26 \%$ compared with control [13]. Despite irrefutable evidence of its benefit, OAC treatment in AF patients is still underused across different parts of the world [14].

The study regarding variation of demographic profile, etiology and usage of anticoagulants in patients with AF is rare in Nepal in contrary to excess disease burden seen in our country. Hence this study was done to emphasize the scenario seen in our context on this regard.

\section{Methods}

A hospital based cross sectional study was carried out in the Department of Car- 
diology of Manmohan Cardiothoracic Vascular and Transplant Center, Institute of Medicine, Kathmandu, Nepal from June 2017 to October 2018. All patients with atrial fibrillation who presented to emergency or had been admitted for other causes and developed AF were enrolled in the study. It was approved by Institutional Review Board of Institute of Medicine. With the written informed consent, a detailed history was taken and physical examination was done. The age, sex, address, underlying comorbidities such as Diabetes Mellitus, Hypertension, Chronic Obstructive Pulmonary Disease, history of smoking, alcohol intake were noted. A twelve lead electrocardiography (ECG) was done and echocardiography with GE, Vivid 7 was done to look for left atrium (LA) size, left ventricular systolic dysfunction, diastolic dysfunction, left ventricular wall thickness, valvular morphology, stenotic or regurgitant lesions. Laboratory investigations included baseline tests with thyroid function test and prothrombin time and international normalized ratio who were already on anticoagulant. The CHA2DS2VASc score was calculated for NVAF. The use of anticoagulants (Warfarin or novel oral anticoagulants such as Dabigatran) or antiplatelets (Aspirin or clopidogrel) among all patients were noted. Bleeding episodes or thromboembolic episodes were noted whether in the past or during the hospital admission. The patients were followed at least for 7 days or till the discharge from the hospital. Statistical data analysis was performed using SPSS version 19.0. Categorical data were expressed as frequencies and percentages.

\section{Results}

A total of 175 patients with atrial fibrillation were included in the study period. The mean age of study patients was 60 years with standard deviation of \pm 16.79 , ranging from minimum of 15 years to maximum of 92 years. Among valvular AF, the mean age was $47.9 \pm 14.013$ and among non-valvular AF it was $67.85 \pm$ 13.507 years.

The female patients were found to be predominant i.e. $56 \%$ whereas $44 \%$ were male patients. The female patients seemed to suffer AF at an earlier age (57.17 \pm 17.84 years) than male patients (63.82 \pm 14.63 years). Seventy five percent of patients with valvular AF were females whereas $56 \%$ of NVAF patients were males.

Majority of patients i.e. $60 \%$ were from outside Kathmandu valley, the capital city. Among total patients, the number of patients with nonvalvular AF was higher i.e. $62 \%$ than valvular AF in our center.

The various etiologic factors of Atrial Fibrillation among study patients showed that the majority 68 (38.9\%) had Rheumatic heart disease [Table 1]. RHD was more common in the age group 46 - 60 years however the youngest was 15 years. Dilated cardiomyopathy (DCM), hypertension (HTN) and degenerative valvular heart disease (DVHD) were common above 60 years [Table 2]. RHD and hypertension were most common among females whereas DCM, Coronary Artery Disease (CAD), DVHD and Mitral valve prolapse (MVP) were more common in males [Table 3]. 
Table 1. Etiological profile of atrial fibrillation.

\begin{tabular}{|c|c|}
\hline Etiology of Atrial fibrillation & Percentage (\%) \\
\hline Rheumatic heart disease & 38.9 \\
\hline Dilated cardiomyopathy & 16.6 \\
\hline Hypertension & 9.7 \\
\hline Degenerative valvular heart disease & 9.1 \\
\hline Acute coronary syndrome & 7.4 \\
\hline Congenital heart disease & 5.7 \\
\hline Ischemic cardiomyopathy & 2.9 \\
\hline Idiopathic & 2.9 \\
\hline Mitral valve prolapsed & 1.1 \\
\hline Hypertrophic cardiomyopathy & 1.1 \\
\hline Chronic obstructive pulmonary disease & 1.1 \\
\hline Alcohol binge & 0.6 \\
\hline Left atrial myxoma & 0.6 \\
\hline Myocarditis & 0.6 \\
\hline Wolff-Parkinson-White syndrome & 0.6 \\
\hline Sick sinus syndrome & 0.6 \\
\hline Hyperthyroidism & 0.6 \\
\hline
\end{tabular}

Table 2. Various etiologies among different age groups.

\begin{tabular}{ccccccc}
\hline \multirow{2}{*}{ Diagnosis } & \multicolumn{5}{c}{ Age group } & Total \\
\cline { 2 - 5 } RHD & $15-30$ & $\mathbf{3 1 - 4 5}$ & $\mathbf{4 6 - 6 0}$ & $\mathbf{6 1 - 7 5}$ & More than $\mathbf{7 5}$ & \\
\hline DCM & 13 & 13 & 29 & 12 & 1 & 68 \\
Congenital HD & 0 & 0 & 9 & 12 & 7 & 29 \\
CAD & 0 & 1 & 5 & 4 & 1 & 10 \\
ICM & 0 & 0 & 0 & 2 & 3 & 13 \\
HTN & 0 & 0 & 3 & 8 & 6 & 17 \\
DVHD & 0 & 0 & 1 & 5 & 10 & 16 \\
MVP & 0 & 0 & 0 & 1 & 1 & 2 \\
HCM & 0 & 0 & 2 & 0 & 0 & 2 \\
Miscellaneous & 0 & 3 & 1 & 2 & 2 & 8 \\
Idiopathic & 0 & 1 & 2 & 0 & 2 & 5 \\
Total & 14 & 18 & 55 & 52 & 36 & 175 \\
\hline
\end{tabular}

Among total patients, 26 (15\%) had Diabetes Mellitus, 3 (1.7\%) had hyperthyroidism, 27 (15.4\%) had hypothyroidism, 50 (29\%) were alcohol consumer and $66(38 \%)$ were smoker. 
Table 3. Various etiologies with gender distribution.

\begin{tabular}{cccc}
\hline \multirow{2}{*}{ Diagnosis } & \multicolumn{2}{c}{ Gender } & Total \\
\cline { 2 - 3 } & Female & Male & 68 \\
RHD & 51 & 17 & 29 \\
DCM & 8 & 21 & 10 \\
Congenital HD & 5 & 5 & 13 \\
CAD & 4 & 9 & 5 \\
ICM & 2 & 3 & 17 \\
HTN & 12 & 5 & 16 \\
DVHD & 7 & 9 & 2 \\
MVP & 0 & 2 & 2 \\
HCM & 1 & 1 & 8 \\
Miscellaneous & 6 & 2 & 5 \\
Idiopathic & 2 & 3 & 175 \\
Total & 98 & 77 & \\
\hline
\end{tabular}

The most common manifestation was dyspnea (69.7\%) with orthopnea (32.5\%) followed by palpitation (19\%) [Table 4].

Around $77 \%$ cases who had thromboembolic events including stroke, lower limb ischemia, renal infarct or splenic infarct had valvular AF and rest $23 \%$ cases had non valvular AF (Dilated cardiomyopathy, systemic hypertension, mitral valve prolapse and ischemic cardiomyopathy). Of 22 thromboembolic events, stroke (63.6\%) was the most common. Among total thromboembolic events, $41 \%$ had predominant mitral stenosis and $23 \%$ had predominant mitral regurgitation.

Among patients with valvular AF, most (79\%) were anticoagulated with warfarin whereas few $(21 \%)$ received antiplatelet therapy. Among nonvalvular AF, $83 \%$ received antiplatelet drugs and $17 \%$ received anticoagulants (mostly Warfarin except two who received Dabigatran and one who received Nicoumalone). The relation of use of anticoagulants in NVAF according to CHADSVasc score was not statistically significant $(\mathrm{p}=0.545)$, which means only few patients were appropriately anticoagulated according to the scores [Table 5].

There were 51 (29\%) patients who had new onset AF among which 7 had valvular AF whereas rest 44 had nonvalvular AF. There were 42 patients with paroxysmal $\mathrm{AF}$ among the new onset $\mathrm{AF}$. The mean age of patients having paroxysmal AF was $63.81 \pm 17.219$ years. The common cause of paroxysmal AF was hypertension (19\%), DVHD (11.9\%), Idiopathic (11.9\%), RHD (9.5\%), DCM (7.2\%), Myocardial Infarction (9.4\%). Other causes were Atrial Septal Defect (ASD), Ventricular Septal Defect (VSD), Alcohol binge, WPW, LA myxoma, Hypertrophic cardiomyopathy (HCM) and other miscellaneous causes [Table 6]. 
Table 4. Clinical manifestations of patients with AF.

\begin{tabular}{cc}
\hline Clinical Features & No. of patients \\
\hline Dyspnea & 122 \\
Orthopnea & 57 \\
Palpitation & 33 \\
Cough & 31 \\
Hemoptysis & 6 \\
Syncope & 6 \\
Pedal edema & 31 \\
Abdominal distension & 22 \\
\hline
\end{tabular}

Table 5. Anticoagulant and antiplatelet drugs use according to CHA2DS2Vasc score.

\begin{tabular}{ccccccccc}
\hline & \multicolumn{9}{c}{ CHA2DS2Vasc score } & \multicolumn{2}{c}{ Total no. } \\
\cline { 2 - 7 } of patients
\end{tabular}

Table 6. Etiologies of patients with paroxysmal AF.

\begin{tabular}{|c|c|c|}
\hline & Frequency & Percent\% \\
\hline $\mathrm{DCM}$ & 3 & 7.2 \\
\hline RHD & 4 & 9.5 \\
\hline HTN & 8 & 19 \\
\hline ASD & 1 & 2.4 \\
\hline STEMI & 2 & 4.7 \\
\hline NSTEMI & 2 & 4.7 \\
\hline Myocarditis & 1 & 2.4 \\
\hline Alcohol binge & 1 & 2.4 \\
\hline WPW & 1 & 2.4 \\
\hline PPCM & 1 & 2.4 \\
\hline ICM & 1 & 2.4 \\
\hline DVHD & 5 & 11.9 \\
\hline LA myxoma & 1 & 2.4 \\
\hline Idiopathic & 5 & 11.9 \\
\hline SSS & 1 & 2.4 \\
\hline RHD with MVR & 1 & 2.4 \\
\hline VSD & 1 & 2.4 \\
\hline IHD & 2 & 4.7 \\
\hline $\mathrm{HCM}$ & 1 & 2.4 \\
\hline Total & 42 & 100 \\
\hline
\end{tabular}


Only $16.7 \%$ of the patients with paroxysmal AF were under anticoagulants, rest received antiplatelets.

\section{Discussion}

Atrial fibrillation is the most common arrhythmia encountered in the hospital admissions and a major cause of morbidity and mortality worldwide. The major classification of AF as valvular and nonvalvular; their etiologic factors, clinical profile and the use of anticoagulants among them were studied.

This study showed that the mean age of patients was 60 years which ranged from 15 years to a maximum of 92 years. The mean age of presentation of patients with valvular AF was 48 years and 68 years in case of nonvalvular AF. The patients with valvular AF seem to be younger than nonvalvular AF which is attributed to Rheumatic heart disease which is seen in younger age group in developing countries like ours. The nonvalvular AF includes patients with dilated cardiomyopathy, hypertension, coronary artery disease, degenerative valvular heart disease and the elderly age itself being the risk factor thus predisposes older age group in this category. A study done in India also revealed RHD more common in people below the age of 50 years whereas hypertension and ischemic heart disease in patients above 50 years [15]. Given the decline in the incidence of rheumatic fever and the relatively low prevalence of RHD in United States, the majority of patients in western studies had NVAF so they had majority of patients in elderly age group [16].

In this study, we found female predominance (56\%). The mean age of female patients was lower (57 years) than males (64 years). Among valvular AF, 75\% were females and among NVAF, 56\% were males showing female preponderance in valvular AF and male preponderance in NVAF. Based on Framingham Study data, it showed that men have a 1.5 -fold greater risk of developing AF than women after adjustment for age and predisposing conditions [17]. The reason of female predominance in our study is because RHD, which is the commonest cause of AF found in our study involving mitral valve in the form of mitral stenosis is more common in females. Henceforth, females presented at younger age. Several studies done in our neighboring country India and few studies in Nepal have shown female predominance, the mean age around fifth or early sixth decade and the commonest cause was RHD [15] [18] [19].

Sixty two percent of patients in our study had nonvalvular AF and the remaining $38 \%$ had valvular AF. Overall, RHD was the most common cause of AF, but it is due to the fact that atrial fibrillation is common in DCM, systemic hypertension, congenital heart diseases like ASD, degenerative valve diseases, myocardial infarction, COPD, MVP, Hypertrophic cardiomyopathy (HCM), WPW syndrome etc, the greater combined burden of these conditions led to greater volume of NVAF in our hospital similar to other studies [20].

A prospective registry done in 46 countries found that hypertension was overall the most common risk factor for $\mathrm{AF}$, that ranged in prevalence from $41.6 \%$ in 
India to $80.7 \%$ in Eastern Europe, RHD was present in only $2.2 \%$ of North American patients, but $21.5 \%$ in Africa and $31.5 \%$ in India [21]. Hypertension is associated with impaired left ventricular diastolic filling, increased left atrial pressure and size and altered conduction velocities that trigger AF [22].

Diabetes mellitus has been associated with a 1.4- to 1.6-fold increased risk of AF perhaps mediated by advanced glycosylation end products and autonomic remodeling. We found that $26(15 \%)$ patients in our study had associated Diabetes Mellitus [23]. Acute coronary syndrome was found to be in $7.4 \%$ cases. Myocardial infarction often causes left ventricular dysfunction and heart failure or atrial ischemia which may promote AF [24].

The use of tobacco has been linked to development of AF as a dose-response effect [25]. The alcohol consumption also result in a hyperadrenergic state, impaired vagal tone, and altered atrial conduction properties, the sum of which predispose to AF [26]. In our study 66 (38\%) were smoker and 50 (29\%) were alcohol consumer however only one case had binge alcohol drinking leading to Holiday Heart Syndrome.

The most common clinical presentation of the patients was dyspnea followed by palpitation similar to other studies [15] [18]. The thromboembolism was seen more in valvular AF than NVAF. It was seen more in mitral stenosis than mitral regurgitation. Among NVAF, it was common in those who had low left ventricular systolic function like DCM and ischemic cardiomyopathy; others like hypertension and mitral valve prolapse. The left ventricular systolic impairment is an independent stroke risk factor due to reduced wall motion and stasis of blood in ventricle forming thrombus; whereas mitral stenosis and hypertension form left atrium clot and subsequently thromboembolism [27]. Despite some of them taking anticoagulant, they had thromboembolism because they had lower INR at the time of presentation since they didn't have regular follow up.

Out of total patients, $61 \%$ were taking antiplatelets and rest was taking anticoagulants. It was seen that $79 \%$ of those with valvular AF were under anticoagulants and rest were taking antiplatelets. However, the patients with NVAF after assessing CHADSVasc score, were found to be suboptimally (17\%) anticoagulated. The inadequacy of anticoagulation can be explained by next finding of our study i.e. about $60 \%$ of the total number of patients were residing outside Kathmandu valley where there is difficult to access medical center easily and lack of facilities for regular INR testing due to which they could not be prescribed anticoagulants. It is due to the fact that if the patients happen to have bleeding or complications due to anticoagulant therapy, they neither can have early approach to medical center nor they can afford for it. Also since most of the patients have lack of knowledge and are ignorant about the need to perform the test regularly and the need for adherence of medication as well as drug interactions with various food and other medications and the need of drug dose modification and lack of compliance of the patients even if they are well explained, the treating doctor have reluctance to prescribe anticoagulant that can have ad- 
ditional attribution for it.

This finding is in contrast to developed countries, where we see that the use of oral anticoagulation among patients with a CHADS2 score of $\geq 2$ to be greatest in North America i.e. 65.7\%. The mean time in the therapeutic range was higher in Western Europe and America than Asia and Africa [21]. The risk of stroke and systemic embolism for AF patients taking warfarin appears to be higher in Asians than non-Asians which were compared in the RE-LY, ROCKET AF and ARISTOTLE trials due to poorer quality of anticoagulation control in Asian patients [28].

Our study showed that among all patients, 29\% had new onset AF among whom most had paroxysmal AF which occurred mostly in NVAF patients, predominantly with hypertension similar to a study done in Italy. Most of them received antiplatelets. Despite the high thromboembolic risk, the use of anticoagulants seemed to be low in other studies as well [29] [30].

\section{Limitation of the study:}

This study is not free of limitations as it is a single center study though the center is a referral superspeciality tertiary care center. It included only the inpatients in emergency and wards or intensive care units. The patients from outpatient department couldn't be included. The time in therapeutic range for anticoagulation could not be calculated as there was lack in follow up of many patients.

\section{Conclusion}

There is a huge disparity in clinical profile and usage of anticoagulants between western countries and developing countries like ours. Rheumatic heart diseases predominate in our country due to which the patients with valvular AF were younger and more of females. Thromboembolic events were seen more in valvular AF. Most but unsatisfactory number of patients with valvular AF were anticoagulated while majority with NVAF received antiplatelets despite of higher CHADS2Vasc score. Though some were under anticoagulants, thromboembolism was still seen as INR was not properly maintained as majority of the patients were devoid of approach to health services. Hence, this study clearly portrays the area of predicament to be focused for further improvement.

\section{Conflicts of Interest}

The authors declare no conflicts of interest regarding the publication of this paper.

\section{References}

[1] January, C.T., Wann, L.S., Alpert, J.S., Calkins, H., Cigarroa, J.E., Cleveland Jr., J.C., Conti, J.B., et al. (2014) 2014 AHA/ACC/HRS Guideline for the Management of Patients with Atrial Fibrillation: A Report of the American College of Cardiology/American Heart Association Task Force on Practice Guidelines and the Heart Rhythm Society. Circulation, 130, 199-267. 
[2] Camm, A.J., Kirchhof, P., Lip, G.Y., Schotten, U., Savelieva, I., Ernst, S., et al. (2010) Guidelines for the Management of Atrial Fibrillation: The Task force for the Management of Atrial Fibrillation of the European Society of Cardiology (ESC). European Heart Journal, 31, 2369-2429. https://doi.org/10.1093/eurheartj/ehq278

[3] Lloyd-Jones, D.M., Wang, T.J., Leip, E.P., et al. (2004) Lifetime Risk for Development of Atrial Fibrillation: The Framingham Heart Study. Circulation, 110, 1042-1046. https://doi.org/10.1161/01.CIR.0000140263.20897.42

[4] Andrade, J., Khairy, P., Dobrev, D. and Nattel, S. (2014) The Clinical Profile and Pathophysiology of Atrial Fibrillation. Relationships among Clinical Features, Epidemiology, and Mechanisms. Circulation Research, 114, 1453-1468. https://doi.org/10.1161/CIRCRESAHA.114.303211

[5] Roby, A. and Manojan, K.K. (2014) Etiological and Clinical Profile of Atrial Fibrillation in Clinical Practice. International Journal of Preventive and Therapeutic Medicine, 2.

[6] Zhou, Z. and Hu, D. (2008) An Epidemiological Study on the Prevalence of Atrial Fibrillation in the Chinese Population of Mainland China. The Journal of Epidemiology, 18, 209-216.

[7] Zubaid, M., Rashed, W.A., Alsheikh-Ali, A.A., Almahmeed, W., Shehab, A., Sulaiman, K., Al-Zakwani, I., Alqudaimi, A., Asaad, N. and Amin, H. (2011) Gulf Survey of Atrial Fibrillation Events (Gulf SAFE): Design and Baseline Characteristics of Patients with Atrial Fibrillation in the Arab Middle East. Circulation: Cardiovascular Quality and Outcomes, 4, 477-482. https://doi.org/10.1161/CIRCOUTCOMES.110.959700

[8] Sliwa, K., Carrington, M.J., Klug, E., Opie, L., Lee, G., Ball, J. and Stewart, S. (2010) Predisposing Factors and Incidence of Newly Diagnosed Atrial Fibrillation in an Urban African Community: Insights from the Heart of Soweto Study. Heart, 96, 1878-1882. https://doi.org/10.1136/hrt.2010.206938

[9] Wolf, P.A., Abbott, R.D. and Kannel, W.B. (1987) Atrial Fibrillation: A Major Contributor to Stroke in the Elderly: The Framingham Study. Archives of Internal Medicine, 147, 1561-1564. https://doi.org/10.1001/archinte.1987.00370090041008

[10] Wolf, P.A., Dawber, T.R., Thomas, H.E. and Kannel, W.B. (1978) Epidemiologic Assessment of Chronic Atrial Fibrillation and Risk of Stroke: The Framingham Study. Neurology, 28, 973-977. https://doi.org/10.1212/WNL.28.10.973

[11] Karatasakis, G.T., Gotsis, A.C. and Cokkinos, D.V. (1995) Influence of Mitral Regurgitation on Left Atrial Thrombus and Spontaneous Echocardiographic Contrast in Patients with Rheumatic Mitral Valve Disease. The American Journal of Cardiology, 76, 279-281. https://doi.org/10.1016/S0002-9149(99)80081-2

[12] Negi, P.C., Sondhi, S., Rana, V., Rathoure, S., Kumar, R., et al. (2018) Prevalence, Risk Determinants and Consequences of Atrial Fibrillation in Rheumatic Heart Disease: 6 Years Hospital Based-Himachal Pradesh-Rheumatic Fever/Rheumatic Heart Disease (HP-RF/RHD) Registry. Indian Heart Journal, 70, S68-S73. https://doi.org/10.1016/j.ihj.2018.05.013

[13] Hart, R.G., Pearce, L.A. and Aguilar, M.I. (2007) Meta-Analysis: Antithrombotic Therapy to Prevent Stroke in Patients Who Have Nonvalvular Atrial Fibrillation. Annals of Internal Medicine, 146, 857-867. https://doi.org/10.7326/0003-4819-146-12-200706190-00007

[14] Huisman, M.V., Rothman, K.J., Paquette, M., Teutsch, C., Diener, H.C., Dubner, S.J., Halperin, J.L., Ma, C., Zint, K., Elsaesser, A., Bartels, D.B. and Lip, G.Y. (2015) Antithrombotic Treatment Patterns in Patients with Newly Diagnosed Nonvalvular 
Atrial Fibrillation: The GLORIA-AF Registry, Phase II. The American Journal of Medicine, 128, 1306-1313. https://doi.org/10.1016/j.amjmed.2015.07.013

[15] Bhardwaj, R. (2012) Atrial Fibrillation in a Tertiary Care Institute-A Prospective Study. Indian Heart Journal, 64, 476-478. https://doi.org/10.1016/j.ihj.2012.07.014

[16] Go, A.S., Hylek, E.M., Phillips, K.A., Chang, Y., Henault, L.E., Selby, J.V. and Singer, D.E. (2001) Prevalence of Diagnosed Atrial Fibrillation in Adults: National Implications for Rhythm Management and Stroke Prevention: The Anticoagulation and Risk Factors in Atrial Fibrillation (ATRIA) Study. JAMA, 285, 2370-2375. https://doi.org/10.1001/jama.285.18.2370

[17] Kannel, W.B. and Benjamin, E.J. (2008) Final Draft Status of the Epidemiology of Atrial Fibrillation. Medical Clinics of North America, 92, 17-40. https://doi.org/10.1016/j.mcna.2007.09.002

[18] Dharma, R.V., Rajaneesh. R.M., Srikanth, K., Raj Kumar, P.B., Satya, P.A. and Guru, P.S.S. (2014) To Study the Prevalence and Clinical Profile of Chronic Atrial Fibrillation in Hospitalized Patients. Nitte University Journal of Health Science, 4, $17-20$.

[19] Dhungana, S.P. and Sherpa, K. (2015) Antithrombotic Agents and Risk Profile of Patients with Atrial Fibrillation from Rural Part of Nepal. Journal of Institute of Medicine, 37, 16-21.

[20] Sastry, K.B.R., Kumar, L.S., Anuradha, P., Raj, B. and Afzal, M.A.M. (2016) Clinical Profile and Echocardiographic Findings in Patients with Atrial Fibrillation. International Journal of Scientific and Research Publications, 6, 44-47.

[21] Oldgren, J., Healey, J.S., Ezekowitz, M., et al. (2014) Variations in Cause and Management of Atrial Fibrillation in a Prospective Registry of 15400 Emergency Department Patients in 46 Countries: The RE-LY Atrial Fibrillation Registry. Circulation, 129, 1568-1576. https://doi.org/10.1161/CIRCULATIONAHA.113.005451

[22] Aronow, W.S. (2017) Hypertension Associated with Atrial Fibrillation. The Annals of Translational Medicine, 5, 457-459. https://doi.org/10.21037/atm.2017.10.33

[23] Dublin, S., Glazer, N.L., Smith, N.L., Psaty, B.M., Lumley, T., Wiggins, K.L., Page, R.L. and Heckbert, S.R. (2010) Diabetes Mellitus, Glycemic Control, and Risk of Atrial Fibrillation. The Journal of General Internal Medicine, 25, 853-858. https://doi.org/10.1007/s11606-010-1340-y

[24] Sinno, H., Derakhchan, K., Libersan, D., Merhi, Y., Leung, T.K. and Nattel, S. (2003) Atrial Ischemia Promotes Atrial Fibrillation in Dogs. Circulation, 107, 1930-1936. https://doi.org/10.1161/01.CIR.0000058743.15215.03

[25] Heeringa, J., Kors, J.A., Hofman, A., Van Rooij, F.J. and Witteman, J.C. (2008) Cigarette Smoking and Risk of Atrial Fibrillation: The Rotterdam Study. American Heart Journal, 156, 1163-1169. https://doi.org/10.1016/j.ahj.2008.08.003

[26] Mukamal, K.J., Tolstrup, J.S., Friberg, J., Jensen, G. and Grønbaek, M. (2005) Alcohol Consumption and Risk of Atrial Fibrillation in Men and Women: The Copenhagen City Heart Study. Circulation, 112, 1736-1742. https://doi.org/10.1161/CIRCULATIONAHA.105.547844

[27] Atrial Fibrillation Investigators (1998) Echocardiographic Predictors of Stroke in Patients with Atrial Fibrillation: A Prospective Study of 1066 Patients from 3 Clinical Trials. Archives of Internal Medicine, 158, 1316-1320. https://doi.org/10.1001/archinte.158.12.1316

[28] Chugh, S.S., Havmoeller, R., et al. (2014) Worldwide Epidemiology of Atrial Fibrillation. A Global Burden of Disease 2010 Study. Circulation, 129, 837-847. 
https://doi.org/10.1161/CIRCULATIONAHA.113.005119

[29] Buccelletti, F., Di Somma, S., Iacomini, P., et al. (2013) Assessment of Baseline Characteristics and Risk Factors among Emergency Department Patients Presenting with Recent Onset Atrial Fibrillation: A Retrospective Cohort Study. European Review for Medical and Pharmacological Sciences, 17, 22-27.

[30] Almeida, E.D., Guimarães, R.B., Stephan, L.S., et al. (2015) Clinical Differences between Subtypes of Atrial Fibrillation and Flutter: Crosssectional Registry of $407 \mathrm{~Pa}$ tients. Arquivos Brasileiros de Cardiologia, 5.

https://doi.org/10.5935/abc.20150049 